\title{
Caudal Epidural Steroid Injections
}

\author{
Bentley A. Ogoke, MD
}

The caudal approach to the epidural space was first reported in 1901. Injection of steroids to treat low back pain was introduced in 1952. Caudal epidural steroid injection is a safe, effective technique when performed with appropriate care under fluoroscopic visualization. Caudal epidural injections are associated with inaccurate needle placement when performed blindly in a substantial number of patients, resulting in intravascular injections as well as other complications.

This review will discuss anatomic and technical considerations of caudal epidural injections, along with advantages, disadvantages, complications, and indications.

Keywords: Caudal epidural steroids, sacral hiatus, fluoroscopy, complications
The caudal approach to the epidural space is the earliest known technique for epidural steroid injection or blocks. It was first reported in 1901 (1-3) and did not gain international universal application until 1925, when Viner (4) popularized its use for treating sciatica. It was not until 1952 that a corticosteroid was added to the local anesthetic injectate mixture and used as the now-common epidural steroid injection for specifically acute and chronic pain (5-8). The popularity of the caudal approach to the epidural space in analgesia has waxed and waned over the decades. Though this article is dedicated to epidural steroid injection for treatment of pain syndromes, it is important to acknowledge the initial surge in popularity of caudal blocks for obstetric analgesia, which was followed by reports of the demise of fetuses due to accidental injection of local anesthetic into the craniums of the descending fetal heads. The lumbar approach presumably allowed placement of injectate closer to the desired anatomic lumbar level, though the accuracy of placement into the epidural space is not guaranteed radiologically as can be the case with the caudal approach.

From Pioneer Valley Pain Management \& Palliative Medicine Center, Inc., Springfield, Massachusetts. Dr. Ogoke is medical director of Pioneer Valley Pain Management \& Palliative Medicine Center. Address Correspondence: Bentley Ogoke, MD, 125 Liberty Street Suite 100, Springfield, Massachusetts 01103.

\section{ANATOMICAL CONSIDERATIONS}

In the caudal approach to the lumbar epidural space, there are pertinent anatomic landmarks and facts worth reviewing. The sacrum consists of five embryonic fused vertebrae. It is convex dorsally. The coccyx consists of three to five rudimentary vertebral bones and is triangular, with the base attached to the sacrum. The superior articular base attaches to the apex of the $V$-shaped sacrum at the sacrococcygeal joint bounded by the sacrococcygeal ligament, which extends dorsally in the midline to cover the sacral hiatus. The sacral hiatus is bordered by the sacral cornu laterally. This space is a natural defect in the union of the dorsal midline of the $\mathrm{S} 5$ vertebrae, where it meets the $\mathrm{S} 4$ vertebrae (partial defect). Its floor is the vertebral body of S5. It contains the coccygeal nerve and the filum terminale. The sacrum has two sets of foramen - the four posterior sacral foramina and the four anterior sacral foramina. The lateral attachment of the sacrum is the sacroiliac joint, while superiorly it is attached to the superior articular facet of the L5-S1 facet joints bilaterally, as well as the L5-S1 disc. The sacral canal contains the epidural venous plexus down to the level of S4. It also contains some epidural fat. The termination of the thecal sac varies depending on age, and varies between the lower border of the S1 foramen in adults and the S3 foramen in children. This is of significance when judging the optimum placement of the epidural needle tip and avoiding dural puncture and postdural puncture headache. The average volume of the sacral epidural canal drops in the geriatric population for a similar height and gender. The height of the patient, as well as the vertical dimension of 


\section{Table 1. Predisposing factors to dural punc- ture in caudal epidural steroid in- jections}

Short stature (height less than 5 feet)

Short sagittal dimension of sacrum

Blind injection without flouroscopic guidance

Inexperienced operator

Tip of the needle above the level of the anterior foramen of

$\mathrm{S} 1$ in anteroposterior view

Atypical anatomy within the sacral canal, including

presence of a tethered cord

the sacrum, e.g., short sacrum, is of clinical significance with respect to the risk of dural puncture (Table 1).

The epidural venous plexus is concentrated in the anterior wall of the sacral canal. Clinically noticeable injury to sacral nerve(s) is rare during caudal epidural steroid injection, even with needle advancement to $\mathrm{S} 1$ or $\mathrm{S} 2$. The anterior sacral foramina allow "leakage" for injectate from the sacral canal and epidural space unlike the posterior sacral foramina, which are covered by a tight band of musculature (9). It is necessary in some clinical situations to advance the epidural needle above the S2 foramen level before injection of local anesthetics and deposteroids, especially if rostral flow can be impeded by any anatomic obstruction.

\section{TECHNICAL CONSIDERATIONS}

In patients who have no significant anatomic variation, needle entry into the epidural space is quick and reliable.

\section{Table 2. Potential causes of difficulty enter-} ing the caudal epidural space

Acute angle of sacral dorsal convexity

Inability to identify anatomic landmarks

Severe to morbid obesity blocking radiologic

(flouroscopic) visualization

Deformity of sacral coccygeal area secondary to previous trauma or birth defect

Sealed sacra; hiatus (rare)

Relatively long coccyx with "superior" location of sacral hiatus

Developmental fusion of sacral canal
There are, however, several causes of difficulty entering the epidural space via the sacral hiatus. The most significant is nonavailability of fluoroscopic guidance. Occasionally, injections do not enter the sacral canal or sometimes needles may enter the sacral canal but leave through the neural foramina dorsally, resulting in the deposteroid's eventually not being left in the sacral canal and epidural space. Table 2 illustrates potential causes of difficulty in entering the caudal epidural space.

Multiple reasons for poor technical outcome of caudal epidural injections include an infrequent finding of abnormal curvature of the sacrum with short angulation, often at the mid or lower one third of the sacrum, impairing the advancement of needle or injectate; morbid obesity, leading to difficulty palpating the sacral cornu and/ or poor X-ray visualization; and absent sacral hiatus, reported to be as high as $5 \%$ to $10 \%$. However, it is likely that this $5 \%$ to $10 \%$ estimate is based primarily upon nonflouroscopically guided injections and is therefore misleading data. Blind injections lead to difficulty with placement, traumatic experience for the patient, and increased likelihood of missing the sacral epidural space or even the sacral canal itself. Inexperience is also a factor that leads to poor outcome, as expected. Pre-existing arachnoiditis, whether clinical or subclinical, can lead to prolonged block in caudal epidural steroid injection. Delays as long as 5 to 6 hours can occur and are thought to be secondary to avid binding. Intraosseous injection can occur when the needle tip advances into the sacral body (modified vertebral body). This occurs more often in nonfluoroscopic-guided injections, as well as in the elderly when osteoporosis sets in. This in turn can lead to local anesthetic toxicity if injectate contains local anesthetic at significant volume and concentration. Patients may complain of a metallic taste in the mouth, numbness of lips, and apprehension; ventricular dysrhythmias may be present, and conduction disturbances may lead to cardiac arrest and death. Respiratory arrest, dizziness, blurry vision and tinnitus can also occur. Seizures may or may not be preceded by restlessness, agitation and paranoia. Other symptoms of CNS depression may occur, including slurred speech, drowsiness and unconsciousness. Muscle twitching often precedes the onset of tonic-clonic seizures. Fortunately, such effects are mild and rarely lead to seizure or respiratory arrest at the average volume of local anesthetic used today in caudal epidural steroid injections, usually 5 to $20 \mathrm{cc}$ of injectate. Table 3 illustrates some of the factors associated with poor outcome.

The presence of lumbar-level obstruction or canal nar- 


\section{Table 3. Factors contributing to poor out- come with caudal epidural steroid injection}

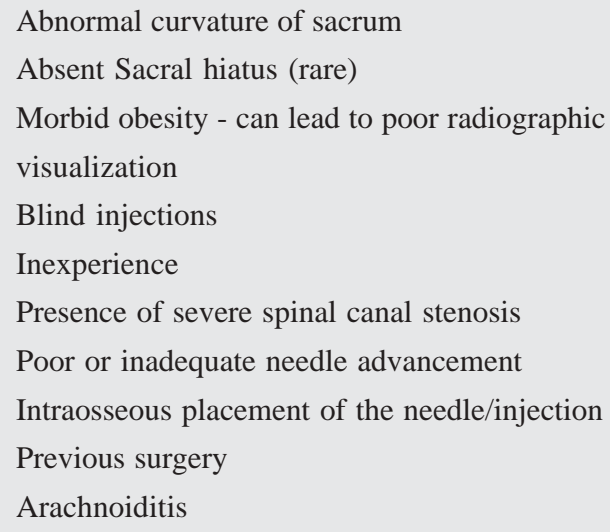

rowing can lead to poor cephalad flow of injectate combined with high injection pressures, as is often noted anecdotally in some spinal canal stenosis patients. In such cases, advancement of the needle to the S1 level may help reduce the leakage from the more distal sacral foramina.

\section{TECHNIQUES OF CAUDAL EPIDURAL STEROID INJECTION}

Caudal epidural steroid injection can be done with the patient prone or in the lateral decubitus position with hip flexed. In view of the location of the sacral hiatus to the anus, strict aseptic technique is required.

The patient is placed in a prone position, and following prepping and draping, the sacral cornu is palpated and the sacral hiatus area infiltrated with local anesthetic at skin level using a $25-\mathrm{G}$ x 1.5 " needle. A lateral decubitus position may also be adopted in patients with colostomy axial spine anomaly or contractures of the extremity, spasticity of trunk with fixed deformity or the presence of ascites or a large abdominal mass. A 45-degree angle entry of the epidural needle should be followed by a radiographic confirmation with anteroposterior and lateral views before advancement. The sacral hiatus opening is visible in the lateral view of fluoroscopy and the sacral coccygeal ligament, when it is being accessed, may feel like a "pop" as the needle enters the ligament. Some practitioners have described this sensation as a sudden loss in resistance after passing through the sacrococcygeal ligament. The needle is then slowly but carefully advanced rostrally. Prior to use of the needle, it might be necessary to pro- duce a convexity at the first 1 inch of the needle (epidural Tuohy needle) and thereafter produce another convexity that is more diffuse throughout the rest of the needle. After puncture of the sacral hiatus, the angle of the entire needle is slowly reduced by bringing the hub of the needle closer to the horizontal as the attempt is made to advance the needle forward. The bevel of the needle at its tip should point upwards if the patient is in prone position or towards the practitioner if the patient is in a lateral position during this initial entry. The tip of the epidural needle during advancement initially is likely to touch the roof of the sacral canal, at which point the needle is then slightly withdrawn by about $2 \mathrm{~mm}$ to $3 \mathrm{~mm}$ and then rotated 180 degrees before being advanced forward slowly to the desired level under fluoroscopic guidance, using both AP and lateral views to confirm needle level and placement. The lateral view is good to confirm that the needle is in the epidural space, while the AP view is good to confirm the actual level at which the tip of the needle is lying relative to the expected location of the thecal sac in the individual patient.

Following placement of the needle, the actual location of the needle is confirmed by loss-of-resistance technique using a puff of air or saline. Before injection of diluted depo-steroid or mixture of local anesthetic and depo-steroid, the needle is aspirated with a 2-cc syringe for blood or CSF. A total of $5 \mathrm{~mL}$ to $25 \mathrm{~mL}$ of injectate is frequently used by most practitioners, with the lower volume reserved for short-statured individuals and the very frail elderly. The larger volume may also be used when the axial spine pathology is located at the lower upper lumbar level. After injection, most patients are recovered supine with vital signs monitored to ensure stability of cardiovascular and respiratory systems. Analgesia is obtained with $0.125 \%$ bupivacaine or $0.5 \%$ lidocaine mixed with 40 to $80 \mathrm{mg}$ of (methylpredrisolone) Depo-Medrol ${ }^{\circledR}$ or 6 to $12 \mathrm{mg}$ of (betamethasone sodium phosphate and betamethasone acetate) Celestone ${ }^{\circledR}$ Soluspan ${ }^{\circledR}$. Other local anesthetics or depo-steroids may also be used $(11,12)$.

\section{ADVANTAGES AND DISADVANTAGES}

Approaches available to access the lumbar epidural space are the caudal, interlaminar, and transforaminal (11-14). The epidural space is compartmentalized into dorsolateral with subdivisions into anterior and posterior $(15,16)$. Even though the caudal epidural injection technique initially enjoyed significant popularity due to the ease of the technique and low incidence of inadvertent dural puncture, it suffered numerous drawbacks secondary to the 
perception of the necessity of injecting a substantial volume of fluid to reach the lumbar nerve roots, lack of agreement as to the exact volume of injection, variation in the anatomy, inaccurate placement of needles in up to $38 \%$ of patients, and inappropriate or uncontrolled spread of anesthetic solution (11-26). However, due to the ease of access and low complication rate, there has been renewed interest in the use of the caudal approach to the epidural space in pain management for administration of local anesthetics, corticosteroids, adhesiolysis, hypertonic saline neurolysis, and spinal endoscopy (11, 12, 22, 27-39).

\section{Advantages}

The advantages of caudal entry into the epidural space include relative ease of entry, and minimal risk of inadvertent dural puncture. Other advantages include that the caudal epidural in steroid injection is the most studied of all three approaches available to access the lumbar epidural space, namely the interlaminar, caudal, and transforaminal; the effectiveness of caudal epidural steroid injection's being superior to interlaminar epidural injection; and the ability to access the epidural space in difficult cases such as postlumbar laminectomy syndrome, as well as the ability to introduce a fiberoptic endoscope into the epidural space through the sacral hiatus.

Available and published evidence is balanced for caudal epidural steroids with regards to effectiveness (11-14, 40, 41), whereas evidence does not support the use of blind interlaminar lumbar epidural steroids (11-14, 40, 41). In a review of 13 trials meeting strict inclusion criteria, five studies involving caudal epidural steroid injections and eight studies involving lumbar epidural steroid injections were reviewed (41). This evaluation showed that, in evaluating the efficiency of caudal epidural injections, four studies were positive, whereas one was negative. However, for lumbar epidural injections, five out of the eight studies showed a negative response. Evaluation of caudal, interlaminar, and transforaminal steroid injections for the management of low back pain revealed surprising results, with cost effectiveness of caudal epidural steroids at $\$ 3,635$ and transforaminal steroids at $\$ 2,927$ per year, whereas interlaminar or lumbar epidural steroids were not shown to be cost effective at a cost of $\$ 6,024$ (40). Essentially the cost-effectiveness studies showed that the cost of blind epidural steroid injections in chronic pain management was higher than percutaneous endoscopic adhesiolysis at $\$ 5,564$ in a heterogenous group of chronic low back pain patients who failed to respond to various other modalities and $\$ 2,028$ in postlumbar laminectomy patients for im- provement of 1 year of quality of life $(33,36)$.

Other advantages of caudal epidural injections include their reported use in anticoagulated patients or patients who suffer with coagulopathy, with increased safety compared to other interlaminar or transforaminal injections. They also have been shown to be advantageous in postlumbar laminectomy patients; as well as in patients suffering with either chemical or compressive radiculitis at the S1 nerve root. The delivery of injectate to S1 nerve roots was seen in $85 \%$ of the patients in one study, indicating the high probability of drugs reaching the involved nerve roots (22). In addition, uncontrolled and anecdotal case reports show that caudal epidural steroid injection is effective for management of pain related to pelvis, rectum, and perineum.

\section{Disadvantages}

The major disadvantages of caudal entry of the epidural space through the sacral hiatus include the necessity of injection of a substantial volume of fluid $(11-14,17,22)$ and unrecognized placement of the needle outside the epidural space in a substantial number of cases (11-14, 17-25). In the early years of the development of caudal epidural injections, Evans (42) in 1930 used large volumes of procaine (up to $140 \mathrm{~mL}$ ) with exceedingly rare occurrences of complications. Cyriax $(43,44)$ also used high-volume injections from 1937 in over 50,000 injections, utilizing $50 \mathrm{~mL}$ of procaine and reported no major disasters but only five temporary complications, with one case of hypersensitivity, two cases of temporary paraplegia of the lower half of the body, and two cases of chemical meningitis; all patients recovered without lasting harm. Bogduk and colleagues $(13,21)$ stated that the reported volume of injectate was $10 \mathrm{~mL}$ to $64 \mathrm{~mL}$ for caudal injection of steroids, and also stated that a volume of $10 \mathrm{~mL}$ should be used to reach the $\mathrm{L} 5$ segment and $15 \mathrm{~mL}$ to reach the L4 segment. Complications have been reported with injections of high volumes into the epidural space, with increases in intraocular pressure with retinal hemorrhage (45-50); however, there are no studies comparing the respective values of different total volumes of either local anesthetic or normal saline for caudal injections. Manchikanti and colleagues (22) assessed the filling patterns of the lumbosacral epidural space by incremental injections of nonionic contrast, and for determination of optimal dose of injectate. They showed that increasing the volume does not necessarily increase the spread into the epidural space or the filling into the nerve roots. In this study (22) epidural spread and nerve-root 
filling increased for nonsurgical and postsurgical patients, for $26 \%$ and $38 \%$ of patients when the injectate was increased from $3 \mathrm{~mL}$ to $6 \mathrm{~mL}$. They also showed that when the injectate was increased from $6 \mathrm{~mL}$ to $10 \mathrm{~mL}$, changes in filling patterns occurred in $38 \%$ of the patients in the nonsurgical group and in $32 \%$ of the patients in the surgical group. However, with an increase from $10 \mathrm{~mL}$ to $15 \mathrm{~mL}$, the increase was seen in fewer than $10 \%$ of the patients in both groups. The filling covering S1 nerve roots was seen in $82 \%$ and $88 \%$ of nonsurgical and postsurgical patients, respectively, indicating the high probability of drugs reaching the involved nerve roots (S1) and significantly lower probability of reaching L5 nerve roots, as filling to the L5 level was seen only in $12 \%$ of the nonsurgical group and 30\% of the surgical group. These authors concluded that increasing the volume of injectate to greater than $10 \mathrm{~mL}$ does not seem to improve the filling pattern.

The second major disadvantage of caudal epidural injections is inaccurate placement of the needle, either extraepidural or intravascular positioning. Inaccurate placement of the needle was reported in $9 \%$ to $38 \%$ of cases when evaluated under fluoroscopic control (11-13, 17-25). Hence, several authors have recommended that any epidural injection be performed using fluoroscopic guidance $(11,12,17-22)$. The advantages of fluoroscopic guidance would include not only improving the accuracy of the needle placement, but also decreasing the risk of a subarachnoid puncture as well as an intrathecal or intravascular injection. White and coworkers (17) showed that incorrect needle placement occurred in approximately $25 \%$ of caudal epidural steroid injections performed by an experienced anesthesiologist and orthopedic surgeon.

Manchikanti and coworkers (22) showed that inaccurate placement occurred in $20 \%$ of patients, with intravascular placement in $7 \%$ and extraepidural placement in $13 \%$. Stitz and Sommer (24) concluded that, while caudal epidural injection is performed ideally with fluoroscopic guidance as the gold standard for accurate drug placement, inaccuracy of blind caudal epidural injection may be reduced by easy identification of anatomic landmarks and absence of palpable subcutaneous air over the sacrum, to $9 \%$. Renfrew and colleagues (20) evaluated caudal epidural steroid injections performed by radiologists and found incorrect needle placement $38 \%$ of the time in experienced hands.

Intravascular uptake or injection of caudal epidural injection was also noted in a significant number of patients.
Renfrew and colleagues (20) observed intravascular uptake in $9 \%$ of the caudal injections. They also reported that no blood was observed returning from the needle on Valsalva maneuver or aspiration. White and coworkers (17) reported 6\% incidence of intravascular uptake. Even though they did not specify how many injections of each approach were performed, they reported that the majority of cases in which intravascular uptake occurred were by the caudal route. They also noted a lack of flashback in many cases of intravascular uptake. Manchikanti and coworkers (22) reported intravascular placement in 7\% of caudal epidural injections without flashback with application of negative pressure and aspiration. Sullivan and colleagues (25) reported an incidence of overall intravascular uptake during lumbar spinal injection procedures as approximately $9 \%$. Even though they did not identify specifically the incidence in caudal epidural injections, they stated that caudal and transforaminal approaches have the highest incidence of intravascular uptake.

The other disadvantages of caudal epidural steroid injections include higher risk of infection, unreliable spread of local anesthetic, patchy blocks, and suitability limited only to lower lumbar and sacral pathology.

\section{COMPLICATIONS}

Infection is a real risk in this procedure. Full and strict aseptic preparations preferably using povidone-iodine $10 \%$ scrub should be adopted in patients not allergic to iodine. Special precaution is indicated in the immunocompromised.

Postinjection pain at the sacral hiatus site of entry may be prolonged but usually does not exceed 2 to 6 months when present. There may be associated injection-site ecchymosis.

Intrathecal injection can occur despite a reduced risk of same and is associated with prolonged and/or high subarachnoid block, respiratory distress or arrest, and total spinal anesthesia with risk of death; adhesive arachnoiditis may result from solvent of depo-steroid polyethylene glycol, and postdural puncture headache may also occur (51). An early sign of intrathecal injection is the patient's inability to evaluate the lower extremity soon after the injection has been completed or the inability to push up the knees in a supine position. Frequently, most patients will complain of feeling numbness in their lower extremities soon after an injection is done, which is not expected 
for an epidural injection.

Nerve injury may occur, but is rare and most likely unrelated to the procedure when it is present. Intravascular or intraosseous injection may lead to toxicity of local anesthetic, e.g., metallic taste in the mouth, oral numbness, dizziness, drowsiness and sedation. Also nausea, seizure and respiratory arrest (apnea) may occur. Bupivacaine tends to produce cardiac toxicity, while lidocaine tends to produce respiratory depression or apnea in higher doses. Urinary retention and incontinence are more a result of use of opioids with local anesthetic than depo-steroid injections.

\section{INDICATIONS}

The most beneficial effects of epidural steroid injection, e.g., caudal epidural steroid injection, at the lumbar level are noted with:

- Annular tear (back sprain),

- Herniated nucleus pulposus with nerve-root irritation,

- Herniated nucleus pulposus without nerve-root compression,

- Chemical neuritis,

- Internal disc disruption syndrome,

- Spondylolisthesis associated with nerve-root irritation,

- Scoliosis with nerve-root entrapment, and

- Spinal stenosis.

There is a possibility that spread of injectate may be hampered or poor with the presence of any significant areas of narrowing of the spinal canal, as in lumbar spinal canal stenosis. Injection pressure may also increase in this scenario.

\section{CONCLUSION}

Chronic low back pain is a major health-care and social problem. Caudal epidural steroid injections are one of the commonly used modalities in managing low back pain. The effectiveness of caudal epidural steroid injections has been demonstrated in multiple studies. Indications for caudal epidural injections include various diagnostic dilemmas, localized neural irritation, and postsurgical syndromes. The knowledge of anatomy, physiology, pharmacology, and advanced technology, along with accurate placement of injectate under fluoroscopic visualization, will effectively improve patient outcomes. However, it is also essential that further meticulously controlled, randomized studies are conducted to prove the rational and unequivocal efficiency of caudal epidural steroid injections.

\section{REFERENCES}

1. Cathelin MF. Mode d'action de la cocaine injecte daus l'escape epidural par le procede du canal sacre. Comptes Rendues des Seances de la Societe de Biologie et de ses Filliales (Paris) 1901; 43:487.

2. Pasquier MM, Leri D. Injection intra- et extradurales de cocaine a dose minime daus le traitments dela sciatique. Bull Gen Ther 1901; 142:196.

3. Sicard MA. Les injections medicamenteuse extradurales par voie saracoccygiene. Comptes Renues des Seances de la Societe de Biologie et de ses Filliales (Paris) 1901; 53:396.

4. Viner N. Intractable sciatica - The sacral epidural injection-An effective method of giving pain relief. Can Med Asso J 1925; 15:630-634.

5. Robechhi A, Capra R. L'idrocortisone (composto F). Prime esperienze cliniche in campon reumatologico. Minerva Med 1952; 98:1259-1263.

6. Lievre JA, Block-Michel H, Attali P. L'injection transscree étude clinique et radiologrique. Bull Soc Med Hosp 1957; 73:1110-1118.

7. Bonica JJ, Backup PH, Anderson CE. Peridural block, an analysis of 3,637 cases. A review. Anesthesiology 1957; 18:723-734.

8. Goebet HW, Jallo SJ, Gardner WJ et al. Painful radiculopathy treated with epidural injections of procaine and hydrocortisone acetate results in 113 patients. Anesth Analg 1961; 140:130-134.

9. Lofstrom B. Caudal anesthesia. In Erickson E (ed). Illustrated Handbook of Local Anesthesia. Copenhagan, Sorrenson and Cohn, 1969, pp 120-129.

10. Tetzlaff JE. Spinal, epidural, and caudal blocks. Clinical anesthesiology. In Morgan GE and Mikhial MS (eds). Appleton and Lange, 1992, p 225.

11. Manchikanti L. The role of neural blockade in the management of chronic low back pain. Pain Digest 1999; 9:166-181.

12. Manchikanti L, Singh V, Bakhit C et al. Interventional techniques in the management of chronic pain: Part 1.0. Pain Physician 2000; 3:7-42.

13. Bogduk N, Christophidis N, Cherry D et al. Epidural Use of Steroids in the Management of Back Pain. Report of Working Party on Epidural Use of Steroids in the Management of Back Pain. Commonwealth of Australia, Canberra National Health and Medical Research Council. 1994, pp 1-76.

14. Weinstein SM, Herring SA, Derby R. Epidural steroid injections. Spine 1995; 20:1842-1846.

15. Hogan QH. Epidural anatomy examined by 
cryomicrotome section. Influence of age, vertebral level and disease. Reg Anesth 1996; 21:295-306.

16. Savolaine ER, Pandya JB, Greenblat SH et al. Anatomy of the human lumbar epidural space: New insights using CT-epidurography. Anesthesiology 1988; 68:217-220.

17. White AH, Derby R, Wynne G. Epidural injections for the diagnosis and treatment of low back pain. Spine 1980; 5:78-86.

18. El-Khoury GY, Ehara S, Weinstein JN et al. Epidural steroid injection: A procedure ideally performed with fluoroscopic control. Radiology 1988; 168:554-557.

19. Stewart HD, Quinnel RC, Dann N. Epidurography in the management of sciatica. Brit J Rheumatol 1987; 26:424-429.

20. Renfrew DL, Moore TE, Kathol MH et al. Correct placement of epidural steroid injections: Fluoroscopic guidance and contrast administration. Amer $J$ Neuroradiol 1991; 12:1003-1007.

21. Bogduk N, Aprill C, Derby R. Epidural steroid injections. In White AH, Schofferman JA (eds). Spinecare: Diagnosis and Conservative Treatment. St. Louis, Mosby, 1995, vol. 1, pp 302-343.

22. Manchikanti L, Bakhit CE, Pampati V. Role of epidurography in caudal neuroplasty. Pain Digest 1998; 8:277-281.

23. Fredman B, Nun MB, Zohar E et al. Epidural steroids for treating "failed back surgery syndrome": Is fluoroscopy really necessary? Anesth Analg 1999; 88:367-372.

24. Stitz MY, Sommer HM. Accuracy of blind versus fluoroscopically guided caudal epidural injection. Spine 1999; 24:1371-1376.

25. Sullivan WJ, Willick SE, Chira-Adisai W et al. Incidence of intravascular uptake in lumbar spinal injection procedures. Spine 2000; 25:481-486.

26. Manchikanti L, Bakhit CE, Pakanati RR et al. Fluoroscopy is medically necessary for the performance of epidural steroids. Anesth Analg 1999; 89:1326-1327.

27. Racz GB, Heavner JE, Diede JH. Lysis of epidural adhesions utilizing the epidural approach. In Waldman $\mathrm{SD}$, Winnie AP (eds). Interventional Pain Management. Philadelphia, WB Saunders, 1996, pp 339351.

28. Racz GB, Holubec JT. Lysis of adhesions in the epidural space. In Racz GB (ed). Techniques of Neurolysis. Boston, Kluwer Academic Publishers, 1989, pp 57-72.

29. Racz GB, Sabonghy M, Gintautas J et al. Intractable pain therapy using a new epidural catheter. JAMA 1982; 248:579-581.

30. Racz GB, Haynsworth RF, Lipton S. Experiences with an improved epidural catheter. Pain Clinic 1986; 1:2127.

31. Racz GB, Heavner JE, Sigleton W et al. Hypertonic saline and corticosteroid injected epidurally for pain control. In Racz GB (ed). Techniques of Neurolysis. Boston, Kluwer Academic Publishers, 1989; pp 73-94.

32. Arthur J, Racz G, Heinrich R et al. Epidural space: Identification of filling defects and lysis of adhesions in the treatment of chronic painful conditions. $A b$ stracts, $7^{\text {th }}$ World Congress on Pain. Paris, IASP Publications, 1993; p557.

33. Manchikanti L, Pakanati RR, Bakhit CE et al. Role of adhesiolysis and hypertonic sailne neurolysis in management of low back pain. Evaluation of modification of Racz protocol. Pain Digest 1999; 9:91-96.

34. Racz GB, Heavner JE, Raj PP. Percutaneous epidural neuroplasty. Prospective one-year follow up. Pain Digest 1999; 9:97-102.

35. Heavner JE, Racz BG, Raj P. Percutaneous epidural neuroplasty. Prospective evaluation of $0.9 \% \mathrm{NaCl}$ versus $10 \% \mathrm{NaCl}$ with or without hyaluronidase. Reg Anesth Pain Med 1999; 24:202-207.

36. Manchikanti L, Pakanati RR, Bakhit CE et al. Nonendoscopic and endoscopic adhesiolysis in post lumbar laminectomy syndrome. A one-year outcome study and cost effectiveness analysis. Pain Physician 1999; 2:52-58.

37. Manchikanti L, Bakhit CE. Percutaneous epidural adhesiolysis. Pain Physician 2000; 3:46-64.

38. Saberski LR, Kitahata LM. Direct visualization of the lumbosacral epidural space through the sacral hiatus. Anesth Analg 1995; 80:839-840.

39. Saberski LR, Brull S. Fiberoptic visualization of the spinal cord. A historical review and report of current methods. Yale Biol Med 1995; 68:7-16.

40. Manchikanti L, Pakanati RR, Pampati V. Comparison of three routes of epidural steroid injections in low back pain. Pain Digest 1999; 9:277-285.

41. Koes BW, Scholten RJPM, Mens JMA et al. Efficacy of epidural steroid injections for low back pain and sciatica: A systematic review of randomized clinical trials. Pain 1995; 63:279-288.

42. Evans W. Intrasacral epidural injection in the treatment of sciatica. Lancet 1930; 2:1225-1229.

43. Cyriax JH. Epidural anesthesia and bedrest in sciatica. Br Med J 1961; 1:20-24.

44. Ombregt L, Bisschop P, Ter Veer JH et al (eds). Treatment of lumbar spine. In Ombregt L, Bisschop P, Ter Veer JH et al (eds). In A System of Orthopedic Medicine. London, WB Saunders, 1995; pp 633-688.

45. Purdy EP, Ajimal GS. Vision loss after lumbar epidural steroid injection. Anesth Analg 1998; 86:119222.

46. Bolder PM, Norton ML. Retinal hemorrhage following anesthesia. Anesthesiology 1984; 61:595-597.

47. Victory RA, Hassett P, Morrison G. Transient blindness following epidural analgesia. Anesthesia 1991; 46:940-941.

48. Stilma JS, de Lange JJ, Crezee FC. Bilateral central scotoma with preservation of central vision in two 
patients following caesarian section under spinal anesthesia. Doc Opthalmol 1987; 67:59-68.

49. Clark CJ, Whitwell J. Intraocular hemorrhage after epidural injection. Br Med J 1961; 2:1612-1613.

50. Kushner FH, Olson JC. Retinal hemorrhage as a consequence of epidural steroid injection. Arch
Ophthalmol 1995; 113:309-313.

51. Nelson DA, Vates TS Jr., Thomas RB Jr. Complications from intrathecal steroid therapy in patients with multiple sclerosis. Acta Neurol Scand 1973; 49:176188. 